\title{
Measuring Wireless Links Capacity
}

\author{
Leopoldo Angrisani*, Alessio Botta ${ }^{\dagger}$, Antonio Pescapé*, and Michele Vadursi ${ }^{\ddagger}$ \\ ${ }^{*}$ Dipartimento di Informatica e Sistemistica, ${ }^{\ddagger}$ Dipartimento di Ingegneria Elettrica, \\ Universitá di Napoli Federico II, 80125 Napoli (Italy) \\ Phone: +390817683170 - Fax: +390812396897 - Email: \{angrisan; pescape; mvadursi\}@unina.it \\ †ITeM Lab, Consorzio Interuniversitario Nazionale per l’Informatica, Napoli (Italy), Email: abotta@napoli.consorzio-cini.it
}

\begin{abstract}
Capacity of a link is a key metric for network design and management. Several tools for capacity measurement are present in the literature and they provide satisfying results when used over wired networks. Their performance over wireless links, however, is not as good, although the techniques they are based on should not suffer from changes at physical layer. This paper accounts for a performance assessment of four tools for capacity measurement, carried out over a wireless link located in a semi-anechoic chamber. The measurement station is designed to guarantee channel stationarity and interference-free measurement conditions, and the performance assessment takes advantage of a proper reference value for the measurand, which is obtained from physical layer measurements. The experimental analysis highlights that the performance of the tools is strongly dependent on the characteristics of the network interface cards that are used, whereas the reference value of capacity, measured at physical layer, does not actually change.
\end{abstract}

\section{INTRODUCTION}

In wireless pervasive computing scenarios a number of applications need to know the value of the available bandwidth as well as the value of the nominal capacity. Network-aware applications (i.e. adaptive streaming applications, adjusting encoding rate applications, ...), peer-to-peer files distribution and applications, applications that need servers selection (i.e. selection of the "best" server in content distribution networks), and scenarios where handoffs are present, are just some of the numerous possible examples.

In wireless networks, such as $802.11 \mathrm{~b}$ WLANs, perceived network performance is influenced by several characteristics typical of underlying wireless network layers, such as modulation schemes, framing procedures, and channel stationary characteristics. Signal integrity in 802.11 b can be strongly degraded due to possible interference from devices of different nature sharing the same band [1], [2], [3]. Since 802.11b WLANs exploit the unlicensed Industrial Scientific Medical (ISM) band, electromagnetic interference comes out to be the most challenging issue in their design and performance evaluation [4]. For these reasons, measuring wireless network Quality of Service (QoS) parameters, and more precisely the channel capacity and its available bandwidth, is not trivial.

Capacity measurements on wireless links are generally less accurate that those performed over wired links [6]. It could be wrong, however, to impute such behavior only to channel non stationarity and/or in-channel interference. To verify that, results provided by widespread measurement tools in a controlled measurement environment are hereinafter analyzed and compared with regard to common metrological indicators, such as $(i)$ measurement repeatability, $(i i)$ difference between reference and measured values of capacity, and (iii) concurrence. The proposed station allows performing measurements at both application and physical layers.

Experimental outcomes confirm that even in an interferencefree environment, and under highly repeatable measurement conditions, the considered tools do not provide as accurate, repeatable, and concurrent results as those achievable over wired links.

The paper is organized as follows. Section II briefly presents the related work; Section III deals with experimental tests, and it is divided into four Subsections that respectively describe the measurement setup, the tools under test, the proposed methodology, and the experimental results; finally, conclusions are drawn in Section IV.

\section{RELATED WORK}

A number of works focused on wireless network capacity and available bandwidth evaluation are present in the literature [5], [6], [7], [8], but, to the best of our knowledge, few works analyze this problem from a metrological point of view. Capacity as well as available bandwidth measurement over wireless links represents an important challenge to be dealt with. For instance, over wireless networks, available bandwidth estimation algorithms that step from the assumption of a stationary channel, fail their mission. Moreover, in literature some papers that present tools aiming to solve this problem do not provide an experimental analysis over wireless networks [5] or, when it is present, the experimental results are not so much satisfying [6].

\section{EXPERIMENTAL TESTS}

The capacity of a network link is defined as the maximum transfer rate achievable at physical layer. The performance of four tools for capacity measurement is evaluated in the following, by analyzing the results of a number of experimental tests on a wireless link located in a controlled, interferencefree, measurement environment. Performance assessment is carried out in terms of repeatability, concurrence of measurement results provided by different tools, and difference between reference and estimated capacity value. To this end, the reference value is properly gained thanks to physical layer measurements, carried out through a Digital Storage Oscilloscope (DSO). 


\section{A. Measurement setup}

Capacity estimations have been performed over the real testbed depicted in Figure 1.

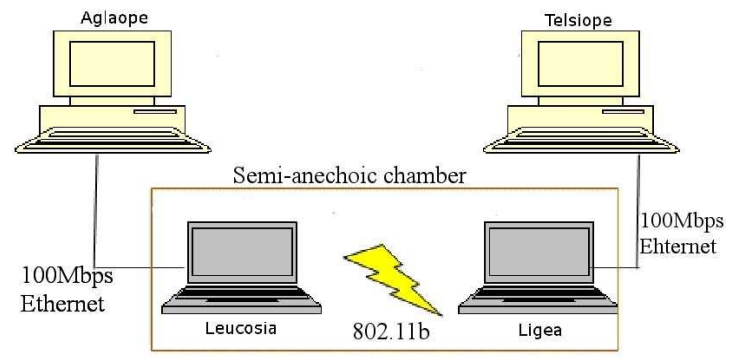

Fig. 1. Experimental testbed

In the testbed, two workstations (named Aglaope and Telsiope) and two laptop computers (Leucosia and Ligea) have been used; all of them were equipped with Linux operating system. The details about the hardware and software configurations are presented in Table I.

TABLE I

TESTBED DETAILS

\begin{tabular}{|c|c|c|}
\hline $\begin{array}{c}\text { Computer } \\
\text { name }\end{array}$ & $\begin{array}{c}\text { Linux } \\
\text { distribution }\end{array}$ & Hardware \\
\hline \hline Aglaope & Debian, & P IV 1.7 GHz Processor, \\
& $2.4 .27-2-386$ kernel & 256MB Ram, 10/100Mbps Eth \\
\hline Telsiope & Debian, & Athlon XP 2.4+ GHz Processor, \\
& $2.4 .27-2-386$ kernel & 256MB Ram, 10/100Mbps Eth \\
\hline Leucosia & Ubuntu Debian, & IBM thinkpad 2682, PIVm 2GHz, \\
& $2.6 .10-5-386$ kernel & 256MB RAM, 10/100Mbps Eth \\
\hline Ligea & Ubuntu Debian, & Toshiba S5200, PIV 2GHz, \\
& $2.6 .10-5-386$ kernel & 512MB RAM, 10/100Mbps Eth \\
\hline
\end{tabular}

As for the wireless Network Interface Cards (NICs), it is worth noting that two different models have been taken into consideration. In particular, two Lucent Orinoco Gold ${ }^{T M}$ 802.11b NICs have been used in the first experimentation stage. Then, the two laptops have been equipped with the D-Link DWL-650 $+{ }^{T M} 802.11 \mathrm{~b}$ NICs. The rate of the link under test has always been fixed to $11 \mathrm{Mbps}$. Moreover, as shown in Figure 1, Leucosia and Ligea have been mandated to forwarding traffic packets from the wired to wireless links. To achieve this goal, on the two laptops the Linux IP_Forward has been activated, and specific routes have been added.

The wireless network has been set up in a semi-anechoic chamber, which prevents from in-channel interference, thus guaranteeing the same operating conditions over successive measurements. This is a fundamental requirement to carry out a significant analysis of measurement repeatability. In order to obtain physical level measurements, the physical signal, captured by a probing antenna, has been analyzed through a wideband DSO (see Table II).

\section{B. Tools under test}

In this work, we have used four tools, named Clink [10], PathRate [11], Pchar [12], and CapProbe [13], to measure the
TABLE II

OTHER TESTBED ELEMENTS SPECIFICATIONS

\begin{tabular}{|c|c|c|}
\hline Equipment & Brand and model & Specifications \\
\hline DSO & Agilent Infiniium & $10 \mathrm{GHz}$ bandwidth, \\
& DSO 81004A & $40 \mathrm{GSample} / \mathrm{s}$ max \\
& & $32 \mathrm{MSample}$ memory \\
\hline Antenna & Electro Metrics EM-6865 & $2-18 \mathrm{GHz}$ frequency range \\
\hline
\end{tabular}

capacity of the link under test. In the following, the techniques on which the four considered tools are based will briefly be introduced. More details can be found in the cited works.

Clink and Pchar are based on the same technique, that is, the Variable Packet Size (VPS). Such technique has been originally proposed by Jacobson in 1997 [14]. It is based on RTT (Round Trip Time) measurements performed on probing packets of different sizes. More details on such technique are presented in Section III-C where the reference value of the measurand is introduced.

The measurement process of Pathrate consists of two consecutive steps. In the first one, the packet pair dispersion is used to obtain the modal distribution of the delay on the path under test. After that, Pathrate performs a packet train dispersion stage in order to estimate the Asymptotic Dispersion Rate (ADR). By combining these two indicators the final capacity estimate is obtained.

Finally, CapProbe is based on packet pair dispersion technique. In the development of this tool, the authors stepped from the consideration that both compression and expansion of packet pair dispersion are due to the queuing effect caused by cross-traffic. For this reason, it estimates the capacity of the end-to-end path by filtering out queuing effects from packet pair dispersion.

\section{Measurement procedure}

A number of experimental tests have been executed to assess the performance of the four considered tools for capacity measurement. In particular, fifty measurements have been carried out for each tool. The estimated value of capacity is then assumed to be the average of all the measurement results, $\bar{C}$. The adopted number of measurements assures satisfying characteristics of the variance of the results [17]. The same number of measurements have been carried out on the opposite path (swapping the roles of source and destination hosts), too. All the tests have also been repeated with the other pair of NICs plugged in. Performance assessment is carried out in terms of $(i)$ repeatability, (ii) difference between reference and estimated capacity value, $\Delta$, and (iii) concurrence of measurement results provided by different tools.

With regard to repeatability, according to the International Vocabulary of Basic and General Terms in Metrology (VIM) [15], it is defined as the closeness of agreement between quantity values obtained by measurements, replicated over a short period of time, under conditions including the same measurement procedure, same operator, same measuring system, same operating conditions and same location. A measure of repeatability is the experimental standard deviation, $\sigma$; the 
lower the experimental standard deviation, the more repeatable the measurement.

Concerning the evaluation of $\Delta$, a proper reference value for capacity is required. In the absence of a more adequate reference, the nominal capacity value could be considered acceptable as a reference value, as it has actually been done in [7]. In this work, a proper reference for the measurand has been drawn from physical layer measurements performed through the DSO. To this end, a typical VPS technique has been utilized. A set of equally spaced packet size values, $L_{1}, L_{2}, . ., L_{N}$, ranging from 70 to 1470 bytes, have been taken into account. For $i=1,2, . ., N, 50$ packets of size $L_{i}$ have been generated, and $\tau_{i, j}$, that is the time the $j$-th packet of size $L_{i}$ occupies the physical channel, is measured taking advantage of the DSO cursors. Then, a linear regression is performed over pairs $\left\{L_{i}, \tau_{i}\right\}$, where $\tau_{i}=\min _{j} \tau_{i, j}$, and the inverse of the slope coefficient is taken as the reference value for capacity, $\tilde{C}$. A different reference is gained for each pair of NICs, as the actual capacity may vary when different NICs are used, in principle.

To generate probing packets, Distributed Internet Traffic Generator (D-ITG) [9] has been used. To capture the packets, a useful feature of D-ITG, already exploited in [16], is used. It consists in the generation of a voltage pulse on the transmitting host serial port each time a packet is generated at application layer; thanks to an EIA-232/TTL converter, such pulse is used as trigger signal for the DSO acquisition. Moreover, to distinguish packets generated by the two hosts, their antennas have been spatially oriented so as to exhibit orthogonal polarization.

Finally, with regard to concurrence analysis, measurement results are expressed in terms of an interval, centered on $\bar{C}$, which is six- $\sigma$ wide. If intervals related to different tools overlap, then measurement results are said to be concurrent.

\section{Results}

As already said in Section III-A, the experimental analysis has been performed by using two different pairs of wireless network interfaces, that are, the Lucent Orinoco Gold ${ }^{T M}$ and the D-Link DWL-G650+ ${ }^{T M}$. In the following, the results obtained by using the first couple will be first presented.In both cases, the experimental results are expressed in terms of mean value, $\mu$, experimental standard deviation, $\sigma$, and difference between reference and estimated capacity value, $\Delta$. As for the reference, by using the methodology explained in section III-C we have obtained two values. The first of them has been achieved by using the Lucent NICs while the second one is related to use of the D-Link NICs. Digging into numerical details, Table III presents the measured reference values.

\section{TABLE III}

REFERENCE CAPACITY VALUES

\begin{tabular}{|c|c|}
\hline Network Interface Cards & Value [Mbps] \\
\hline Lucent Orinoco Gold & 11.007 \\
\hline D-Link DWL-650+ & 11.042 \\
\hline
\end{tabular}

a) Lucent Orinoco NICs: The tools have been first run with default configuration parameter values, carrying out several consecutive measurements, obtaining the outcomes reported in Table IV. Such table shows that measurement results provided by the different tools are much lower than nominal capacity value, besides being not concurrent. Repeatability of measurement results is, however, very good, as $\sigma \%$ is lower than $1 \%$.

TABLE IV

CAPACITY ESTIMATES WITH LUCENT CARDS [MBPS]

\begin{tabular}{|c|c|c|c|c|}
\hline & Clink & Pathrate & Pchar & CapProbe \\
\hline$\mu[\mathrm{Mbps}]$ & 2.8341 & 6.162 & 3.848 & 4.046 \\
\hline$\sigma[\mathrm{Mbps}]$ & 0.0008 & 0.007 & 0.005 & 0.036 \\
\hline$\sigma \%$ & 0.030 & 0.13 & 0.13 & 0.90 \\
\hline$\Delta[\mathrm{Mbps}]$ & 8.1729 & 4.845 & 7.159 & 6.961 \\
\hline$\Delta \%$ & 74 & 44 & 65 & 63 \\
\hline
\end{tabular}

Before performing the tests with the second couple of NICs, other experimentations have been carried out in order to understand why Clink and Pchar, which use the same VPS technique to measure capacity, provide quite different results. To this aim, the tools have been run in debugging mode recording the RTT values they measured for each used packet size. A linear regression on RTT values has then been performed, obtaining the slope of the approximating curve, that is, the reciprocal of the capacity. By comparing such capacity value to that provided directly by the tools it has come out that Clink actually operates a linear regression on RTT values, whereas the filtering technique applied by Pchar is probably responsible for lower capacity estimation.

b) D-Link NICs: As in the previous case, the tools have first been run with default configuration parameter values, carrying out several consecutive measurements. The obtained results are reported in Table $\mathrm{V}$.

TABLE V

CAPACITY ESTIMATES WITH D-LINK CARDS [MBPS]

\begin{tabular}{|c|c|c|c|c|}
\hline & Clink & Pathrate & Pchar & CapProbe \\
\hline$\mu[\mathrm{Mbps}]$ & 7.2715 & 6.264 & 10.591 & 4.819 \\
\hline$\sigma[\mathrm{Mbps}]$ & 0.0023 & 0.011 & 0.030 & 0.046 \\
\hline$\sigma \%$ & 0.030 & 0.17 & 0.28 & 0.96 \\
\hline$\Delta[\mathrm{Mbps}]$ & 3.771 & 4.778 & 0.451 & 6.223 \\
\hline$\Delta \%$ & 34 & 43 & 4.1 & 56 \\
\hline
\end{tabular}

The table shows that, like in the previous case, the results are not concurrent while the repeatability is very good, as $\sigma \%$ is lower than $1 \%$. Also, the capacity estimates are, in some cases, different from those of the previous measurement stage. In particular, by comparing the values reported in Tables IV and $\mathrm{V}$, we observe that:

- very different capacity estimates are provided by both Clink and Pchar. Indeed, $\Delta$ associated to both tools is much lower than that of the previous case. Pchar has, in particular, provided the best results in terms of difference between estimated and reference values of capacity ( $\Delta=$ $4.1 \%)$; 
- little differences are observed in the results of both Pathrate and CapProbe.

All the results show that capacity estimation is very sensitive to the adopted NICs. Moreover, when RTT-based tools (i.e. Clink and Pchar) are used, wireless network interfaces commonly considered more reliable (Lucent Orinoco) exhibit the worst performance. In order to understand this behavior the experimental analysis presented in the next paragraph has been performed.

c) Physical layer measurements: To investigate why capacity estimates based on VPS technique are so inaccurate when performed by using the Lucent Orinoco NICs, physical layer RTT values have been measured through the DSO. In this analysis synthetic traffic has been generated through DITG [9], which has the capability of signaling the departure time of packets through the serial port, thus triggering DSO acquisitions. Once transmitted packets have been captured, two time intervals, $t_{1}$ and $t_{2}$ have been measured. The former represents the time that a packet (plus its MAC level ACK) takes to traverse the medium, whereas $t_{2}$ is equal to $t_{1}$ plus the time elapsed from the end of the MAC layer ACK transmission to the beginning of the response packet.

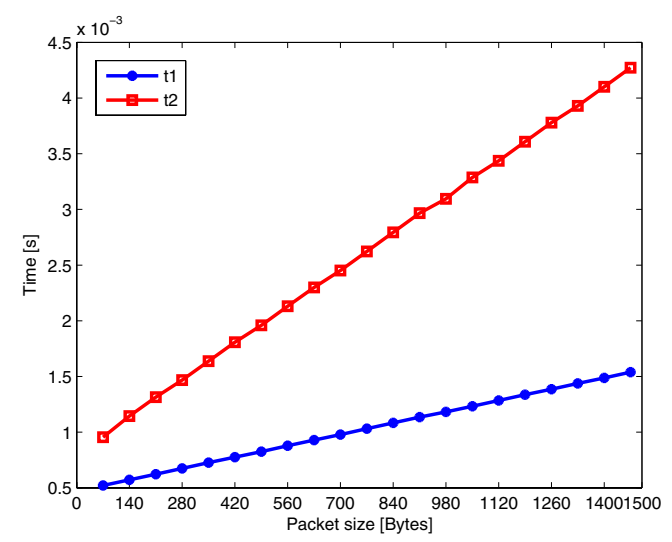

Fig. 2. Measured time intervals vs packet sizes

Fig 2 shows the values of $t_{1}$ and $t_{2}$ as a function of packet size. By operating linear regression over each of the two curves, two different estimates of capacity are gained: $C_{t 1}=11.009 \mathrm{Mbps}$ and $C_{t 2}=3.495 \mathrm{Mbps}$. While $C_{t 1}$ is very similar to the expected nominal capacity value, $C_{t 2}$ represents a significant underestimation. Such results prove that: i) the physical layer capacity respects the 802.11 b standard specifications; ii) wrong results provided by Clink and Pchar are a consequence of the presence of an interval $\delta=t_{2}-t_{1}$ that grows linearly with the packet size with an unexpected rate that causes a significant capacity underestimation. As for this last result, in order to understand the nature of such interval $\delta$, the same physical layer measurement have been performed by using the D-Link NICs.

Figure 3 shows the results of such analysis. In particular, in this figure, $t_{1}$ and $t_{2}$ are sketched as a function of the packet size. The linear regression over the curves provides two different capacity values: $C_{t 1}=11.042 \mathrm{Mbps}$ and $C_{t 2}=$ 8.537Mbps. In contrast with the results presented in Figure 2, the $\delta$ interval induces a much lower capacity underestimation.

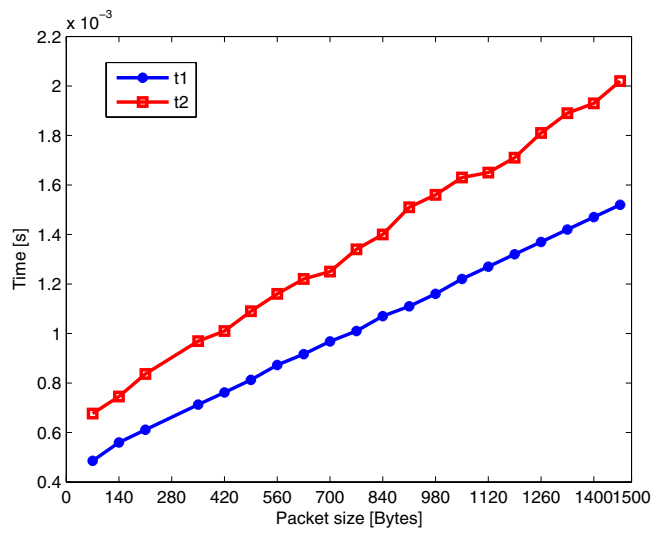

Fig. 3. Measured time intervals vs packet sizes

In conclusion, the experimental analysis allows understanding that the capacity estimation over wireless networks is strongly influenced by wireless network interface cards. In particular, we have found that the used Lucent Orinoco Gold ${ }^{T M}$ NICs cause non negligible estimation errors in the tools that are based on RTT measurement. Nevertheless, experienced capacity estimates are generally inaccurate, no matter which NICs are used, as the difference between reference and estimated value is minor than $5 \%$ in only one case.

\section{CONCLUSiON}

In this work several bandwidth measurements on a real wireless test-bed, situated in a semi-anechoic chamber, have been performed. Such environment allows considering the channel as immune from interference, that is, in optimal and repeatable measurement conditions. Even in such conditions, results provided by tools that perform well in wired scenarios, are significantly different from reference values, and not concurrent, although exhibiting good repeatability ( $\sigma$ always inferior to $1 \%$ ). The experimental analysis has been conducted through different steps, each of which at different TCP/IP layer. Measurement outcomes at physical layer have shown that performance of RTT-based tools strongly depends on the particular NICs hosts are equipped with. In particular, by using Lucent Orinoco NICs, large differences between measured and reference values of capacity have been experienced with RTT-based tools. This is true even if the capacity measured at physical layer is actually compliant to the IEEE $802.11 \mathrm{~b}$ standard with all the considered network interfaces.

\section{REFERENCES}

[1] J. Lansford, A. Stephens, and R. Nevo, "Wi-Fi (802.11b) and Bluetooth: Enabling Coexistence," IEEE Network, Sept./Oct. 2001, vol. 15, pp. 2027.

[2] N. Golmie, N. Chevrollier, O. Rebala, "Bluetooth and WLAN coexistence: challenges and solutions," IEEE Wireless Communications, Vol.10, Iss.6, Dec. 2003, pp. 22- 29. 
[3] Y. Matsumoto, M. Takeuchi, K. Fujii, A. Sugiura, Y. Yamanaka, "Performance analysis of interference problems involving DS-SS WLAN systems and microwave ovens", IEEE Transactions on Electromagnetic Compatibility, Vol.47, Iss.1, Feb. 2005, pp. 45- 53.

[4] K. Takaya, Y. Maeda, N. Kuwabara, "Experimental and theoretical evaluation of interference characteristics between 2.4-GHz ISM-band wireless LANs", IEEE International Symposium on Electromagnetic Compatibility, 1998, Vol.1, 24-28 Aug 1998,

[5] R. Kapoor, L.-J. Chen, A. Nandan, M. Gerla, M. Y. Sanadidi, "CapProbe: A Simple and Accurate Capacity Estimation Technique for Wired and Wireless Environments," ACM SIGMETRICS Performance Evaluation Review, Volume 32, Issue 1, June 2004.

[6] K. Lakshminarayanan, V. N. Padmanabhan, J. Padhye, "Bandwidth Estimation in Broadband Access Networks," Proc. of Internet Measurement Conference 2004

[7] Samarth Shah, Kai Chen, Klara Nahrstedt, "Available bandwidth estimation in IEEE 802.11-based wireless networks," December 2003 - CAIDA BEst workshop

[8] K. Lai, M. Baker, "Measuring Bandwidth," Proc. of Infocom 1999.

[9] http://www.grid.unina.it/software/ITG.

[10] A.B. Downey, "Using Pathchar to Estimate Internet Link Characteristics," Proc. of ACM SIGCOMM, Sept. 1999, pp.222-223.

[11] C. Dovrolis, P. Ramanathan, D. Moore, "What do packet dispersion techniques measure?," Proc. of Infocom 2001.

[12] B. A. Mah, "pchar: a Tool for Measuring Internet Path Characteristics," Feb.1999, http://www.employees.org/_bmah/Software/pchar/.

[13] R. Kapoor, L-J. Chen, L. Lao, M. Gerla, and M. Y. Sanadidi, CapProbe: A Simple and Accurate Capacity Estimation Technique, Proc. of SIGCOMM 2004

[14] V. Jacobson. "pathchar - a tool to infer characteristics of Internet paths", 1997. Presented as April '97 MSRI talk.

[15] International Standard Organization (ISO), "International Vocabulary of Basic and General Terms in Metrology (VIM)," Third Edition, draft paper, Geneva, September 2004.

[16] L. Angrisani, S. D'Antonio, M. Esposito, M. Vadursi, "Techniques for Available Bandwidth Measurement in IP Networks: a Performance Comparison," accepted for publication, Computer Networks (Elsevier).

[17] International Standard Organization (ISO), Guide to the expression of uncertainty in measurement, 1993. 